\title{
A ROBUST OPEN-CLOSE SEQUENCE FILTER BASED ON MATHEMATICAL MORPHOLOGY
}

\author{
Shyam Lal', Akhilesh Shukla ${ }^{2}$, Amit Sharma ${ }^{3}$, Pradeep Gupta ${ }^{4}$, Sanjay Kumar ${ }^{5}$ \\ Department of Electronics \& Communication Engineering, \\ Moradabad Institute of Technology, Moradabad, U.P, India \\ E-mail : 'shyam_rao24@rediffmail.com, ${ }^{2}$ manu123@yahoo.com,
}

\begin{abstract}
A robust open close sequence filter based on mathematical morphology for high probability impulse noise removal is presented. First, an impulse noise detector using mathematical residues is proposed to identify pixels that are contaminated by the salt or pepper noise. Then the image is restored using specialized open-close sequence algorithms that apply only to the noisy pixels. Finally, black and white blocks that degrade the quality of the image will be recovered by a block smart erase method. Simulation and experimental results demonstrate that the proposed robust open close sequence filter outperforms a number of existing algorithms and is particularly effective for the very highly corrupted images.
\end{abstract}

Key words: Dilation, Erosion, Opening, Closing, Median filter, CWM Filter and PWMAD Filter

\section{INTRODUCTION}

The goal of removing impulse noise is to suppress the noise while preserving the integrity of edge and detail information associated with the original image. To this end, nonlinear techniques have been found to provide more satisfactory results in comparison with linear methods. The traditional median filters process all the pixels of the image, without taking account of whether or not a pixel is corrupted by impulse noise [1]. To avoid this disadvantage, it is important to detect whether the current pixel is corrupted. These approaches involve a preliminary identification of corrupted pixels in an effort to prevent alteration of true pixels. Among those are center weighted median (CWM) filter [2-4], pixel-wise MAD (PWMAD) [5$6]$, and recently, impulse noise removal based on mathematical morphology has been attracting research effort [7-12]. Mathematical morphology is nonlinear image processing methodology. After that, mathematical morphology has become popular in the image processing field, due to its rigorous mathematical description and its proven applicability in a number of imaging problems, including noise elimination, feature extraction, and image compression[13-16]. To remove the commonly found noise without losing details of the image, morphological filters with directional, multiple structuring elements, or multiscale filtering, have been used. Rank-order filter using impulse noise detection has also been successfully applied. Although the above filters give a better performance in terms of impulse noise removal, they still tend to remove details from the image or retain too much impulse noise in extremely high impulse occurrence (e.g., $\%)$. In this paper, we propose a robust open-close sequence (OCS) filter to restore images that are corrupted by $30 \%-80 \%$ probability impulse noise.

\section{BASIC BACKGROUND OF MATHEMATICAL MORPHOLOGY}

Mathematical morphology is nonlinear image processing methodology that is based on the application of lattice theory to spatial structures. After that, mathematical morphology has become popular in the image processing field, due to its rigorous mathematical description and its proven applicability in a number of imaging problems, including noise elimination, feature extraction, and image compression [13-16].

Two fundamental mathematical morphological operations are: dilation and erosion. In fact, many of the morphological algorithms are based on these two primitive operations. In gray scale images, we develop algorithms for boundary extraction via a morphological gradient operation, and for region partitioning based on texture content. We operation are also useful in smoothing and sharpening, which often are useful as per or post processing steps [13-16].

\section{A. Dilation}

If $A$ and $B$ as sets in $Z^{2}$, the dilation of $A$ and $B$, denoted $\mathrm{A} \oplus \mathrm{B}$, is defined as

$$
A \oplus B=\{z \mid(B) z \cap A \neq \phi\}
$$

This equation is based on obtaining the reflection of $B$ about its origin and shifting this reflection by $z$. the dilation of $A$ and $B$ then is the set of all displacement, $z$, such that $B$ and $A$ overlap by at least one element. Gray scale dilation of $f$ by $b$, denoted $f \oplus b$, is defined as

$$
(f \oplus b)(s, t)=\max \left\{f(s-x, t-y)+b(x, y) \mid(s-x),(t-y) \in D_{i} ;(x, y) \in D_{b}\right\}
$$


where $D_{f}$ and $D_{b}$ are the domains of fand $b$, respectively.

\section{B. Erosions}

If $A$ and $B$ as sets in $Z^{2}$, the erosion of $A$ and $B$, denoted $A \Theta B$, is defined as

$$
A \Theta B=\left\{z \mid(B)_{z} \subseteq A\right\}
$$

This equation indicates that the erosion of $A$ and $B$ is the set of all points $z$ such that $B$, translated by $z$, is contained in $A$. Gray scale erosion of $f$ by $b$, denoted $\oplus \oplus b$, s defined as

$(f \Theta b)(s, t)=\min \left\{f(s+x, t+y)-b(x, y) \mid(s+x),(t+y) \in D_{f} ;(x, y) \in D_{b}\right\}$

where $D_{f}$ and $D_{b}$ are the domains of $f$ and $b$, respectively.

\section{Opening}

Opening of image $f$ by sub image (structuring element) $b$, denoted $\mathrm{fob}^{\mathrm{b}}$ is

$$
f \circ b=(f \Theta b) \oplus b
$$

Opening is simply the erosion of $f$ by $b$, followed by a dilation of the result by $b$. The opening simplifies by removing the bright components that do not fit within the structuring element.

\section{Closing}

Closing of image $f$ by sub image (structuring element) $b$, denotedf $\mathrm{is}$

$$
f \bullet b=(f \oplus b) \Theta b
$$

closing is simply the dilation of $f$ by $b$, followed by a erosion of the result by $b$. The closing simplifies by removing the dark components that do not fit within the structuring element.

\section{PROPOSED OPEN-CLOSE SEQUENCE FILTER}

The proposed open-close sequences (OCS) filter to restore images that are corrupted by $40 \%-80 \%$ probability impulse noise. The scheme of the proposed filter is shown in Fig 1 .

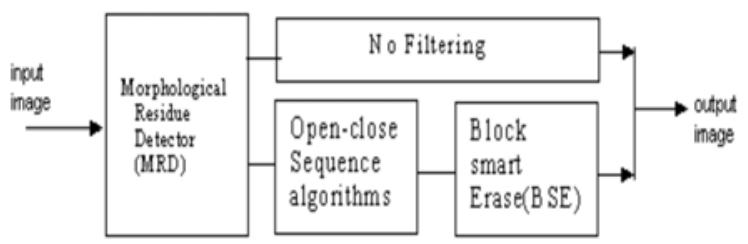

Figure.1. Open - Close sequence (OCS) filter
Main components of the proposed open close sequence (OCS) filter are as follows:-

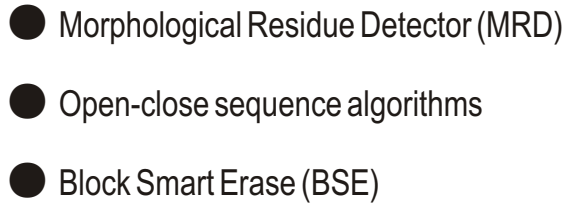

In this section, we start by describing the noise detector based on morphological residues. Then we introduce the open-close sequence algorithms and block smart erase method. Finally, the open-close sequence filter is proposed.

\section{A. Morphological Residue Detector (MRD):}

In this method, we detect noisy pixels using mathematical morphology. As usual, erosion outputs the minimum value in the structuring element domain, and dilation outputs the maximum value in the structuring element domain. Therefore, the opening removes salt impulse noise, and the closing removes pepper impulse noise. The determination of the salt or pepper noises is easily done by comparing the difference between the value of the pixel and the result of opening and closing with a flat structuring element [17-18]. Let $D_{0}$ and $D_{c}$ be the opening and closing absolute distance from input signal respectively

$$
\begin{aligned}
& D_{o}=f-f \circ b \\
& D_{c}=f \bullet b-f
\end{aligned}
$$

Here $D_{o}$ and $D_{c}$ are nonnegative. Noise pixels are detected by comparing $D_{0}$ and $D_{c}$ with a threshold $T$.

$$
r(i, j)= \begin{cases}1 & D_{o} \geq T \text { and } D_{c}=0 \\ -1 & D_{o}=0 \text { and } D_{c} \geq T \\ 0, & \text { otherwise }\end{cases}
$$

If ${ }^{r(i .)}$ is 1 , then is regarded as salt noise; if ${ }^{r(i)}$ is -1 , then is regarded as a pepper noise; otherwise, if ${ }^{r(i)}$ is 0 , then ${ }^{r(i .)}$ is regarded as an original pixel. This detector uses two parameters: the threshold T and the size of the structuring element $b$. It is easy to optimize the parameters by means of experiment. Whenever these noises are not or cannot be detected, the pixel value is considered noiseless, and it is passed thorough. When a certain noise is detected, the corresponding generalized open-close sequence algorithm is selected; otherwise, the input signal is put forward [17-18]. 


\section{B. Open-Close Sequence Algorithm:}

Two filters using open-close sequences are employed to the detected impulse noise. The first one called open-close filter (OCF) is defined as follows:

$$
O C F(f)=\left(f \circ b_{1}\right) \bullet b_{2}
$$

Multiscale structure elements are introduced to the open-close sequence algorithm. The size $b_{1}$ of must be small enough to preserve more details of the image, and the size of $b_{2}$ is larger than that of $b_{1}$. The opening efficiently eliminates the detected salt noises, but at the same time, the pepper noises are magnified by the first erosion. So it is necessary to use closing to remove the additional pepper noises. However, infected pepper noises whose sizes exceed that of $b_{1}$ cannot be eliminated. To achieve better performance, the scale of $b_{2}$ must be much larger than that of $b_{1}$. It is appropriate that the size $b_{1}$ of is $5 \times 5$ (or $7 \times 7$ ), which is not so large as to remove lots of image details. Then the pepper noise can be eliminated powerfully by the following closing while more image details can be preserved. The second sequence called close-open filter (COF) is defined as follows

$$
\operatorname{COF}(f)=\left(f \bullet b_{1}\right) \circ b_{2}
$$

Corresponding to the first filter, the second one applied to remove the detected pepper noises consists of one closing and one opening. Both OCF and COF filters are combinations of opening and closing operators and perform efficiently to remove the corresponding impulse noise. However, the noises whose size is larger than the size of $b_{1}$ will not be eliminated and propagated in the image. The filtered image appears to have some uncomfortable characteristics: undesirable white (or black) blocks are generated during the OCF (or COF) procedure and reserved in the filtered result. Their sizes are large enough and cannot be eliminated by above two sequence filters [17-18].

\section{Block Smart Erase Algorithm}

The existence of white and black blocks degrades the filtered image significantly and makes the filtered image look uncomfortable. A simple and efficient algorithm called block smart erase (BSE) algorithm is proposed to eliminate their effects. The BSE algorithm is based on median technology and takes the place of the extreme value (black or white) pixel by the median value of their surrounding pixels [17-18]. The details of the proposed scheme are shown as follows.
1) For an $\mathrm{NxN}$ window centered at the test pixel, where $\mathrm{N}$ would normally be $5,7,9, \ldots \ldots$ and larger value should be suggested.

2) $\mathrm{If}=0$ or $=255$, is an absolute extreme value pixel that must be estimated; go to step 3. Otherwise, the value of is not altered; go to step 4.

3) When an extreme value pixel is detected, its gray level is substituted by the median value of the window.

4) The procedure is repeated for the next window.

It can be seen that the white and black blocks are efficiently removed. The OCS filter can be defined as

$$
\operatorname{OCS}(f)=\frac{B S E_{w}(O C F(f))+B S E_{b}(C O F(f))}{2}
$$

\section{SIMULATION RESULTS}

The 8-bit images of dimensions MIxM2 (= 256x256) pixels is used for simulations. The pixels $\mathrm{s}(\mathrm{i}, \mathrm{j})$ for $1 \nless \varangle \mathrm{M} 1$ and $1 \Varangle \varangle \mathrm{M} 2$, of the image is corrupted by adding impulse noise, with noise density ranging from 0.1 to 0.8 . In all the simulations, square windows of dimensions $\mathrm{NxN}$ pixels and with different values of width $N(=3,5,7)$ are used. The Peak signal to noise ratio (PSNR) is used to compare the relative filtering performance of various filters. The PSNR between the filtered output image $y(i, j)$ and the original image $s(i, j)$ of dimensions MIxM2 pixels is defined as:

$$
P S N R=20 * \log _{10}\left(\frac{M A X_{t}^{2}}{\sqrt{M S E}}\right)
$$

Where ${ }^{\text {MAX }}$ is max pixel value of the image, and MSE is defined as

$$
M S E=\frac{\sum_{i}^{M L^{2}} \sum_{j}^{2}[y(i, j)-s(i, j)]^{2}}{M 1 \times M 2}
$$

It can be seen that Peak signal to noise ratio (PSNR) is closely related to mean square error (MSE). 
Table 4.1 PSNR values for different filters on 'Lenna' image

\begin{tabular}{|l|l|l|l|l|l|l|l|l|}
\hline Filter & \multicolumn{7}{|c|}{ Noise Density } \\
\cline { 2 - 9 } & $10 \%$ & $20 \%$ & $30 \%$ & $40 \%$ & $50 \%$ & $60 \%$ & $70 \%$ & $80 \%$ \\
\hline Median & 29.8 & 26.8 & 22.3 & 18.3 & 14.8 & 12.0 & 9.67 & 7.86 \\
\hline CWM & 30.4 & 27.4 & 23.0 & 19.2 & 15.5 & 12.6 & 10.7 & 8.23 \\
\hline PWMAD & 28.76 & 27.6 & 26.1 & 24.0 & 20.8 & 18.0 & 14.7 & 11.1 \\
\hline OCS & 25.85 & 25.1 & 24.6 & 23.7 & 22.5 & 21.2 & 19.9 & 17.7 \\
\hline
\end{tabular}

Table 4.2 PSNR values for different filters on 'House' image

\begin{tabular}{|l|l|l|l|l|l|l|l|l|}
\hline \multirow{2}{*}{ Filters } & \multicolumn{7}{|c|}{ Noise Density } \\
\cline { 2 - 9 } & $10 \%$ & $20 \%$ & $30 \%$ & $40 \%$ & $50 \%$ & $60 \%$ & $70 \%$ & $80 \%$ \\
\hline Median & 31.03 & 27.32 & 22.69 & 18.38 & 14.46 & 11.85 & 9.43 & 7.52 \\
\hline CWM & 31.96 & 27.77 & 23.57 & 19.22 & 15.82 & 12.70 & 10.06 & 7.90 \\
\hline PWMAD & 30.81 & 29.28 & 27.95 & 24.72 & 21.14 & 18.30 & 14.78 & 11.30 \\
\hline OCS & 26.01 & 25.22 & 24.49 & 25.47 & 22.29 & 20.80 & 19.48 & 16.86 \\
\hline
\end{tabular}

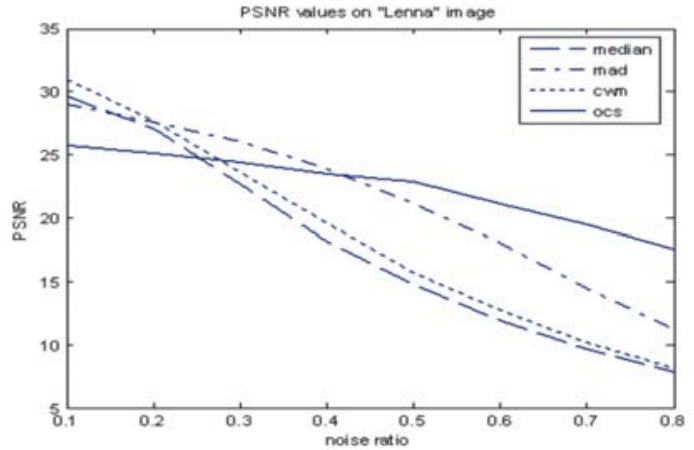

Fig 2.(a) PSNR value on "Lenna" Image PSNR values on "House" image

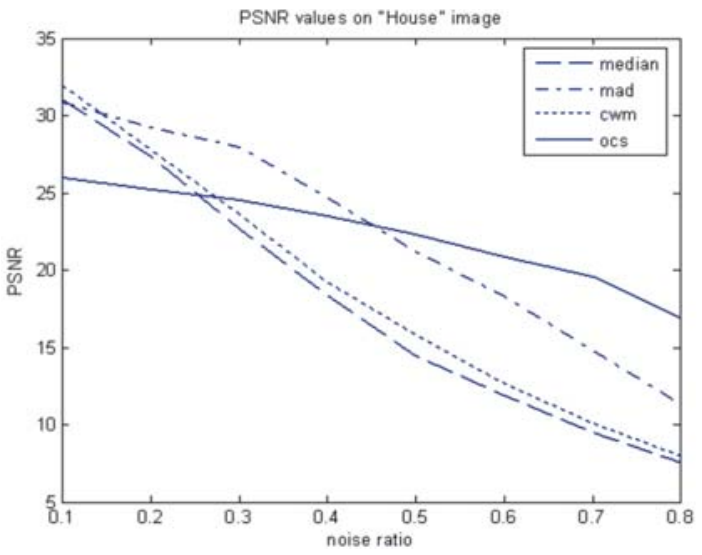

Fig 2.(b) PSNR Value on "House" Image

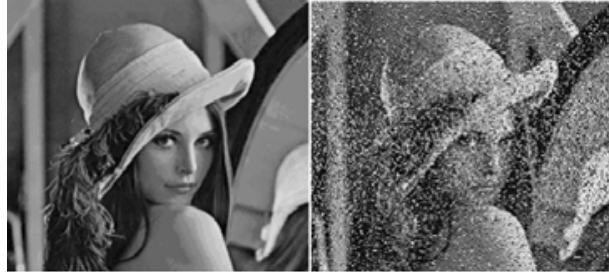

Fig 3.(a)Origanal Image(b) Corrupted With 30\% Noise

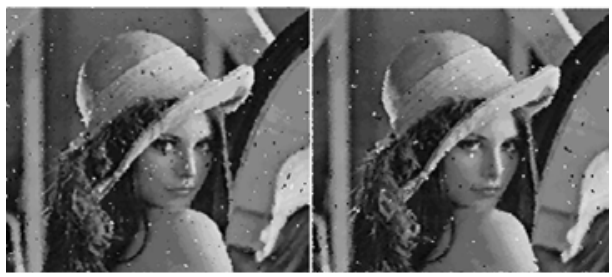

Figure 3.(c) Output from median filter (d) Output from cwm filter

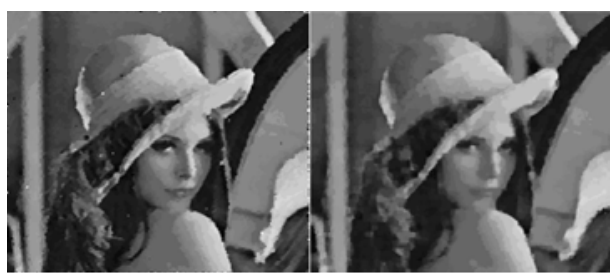

Fig 3.(e)Output from Pwmad Filter (f) output From OCS Filter

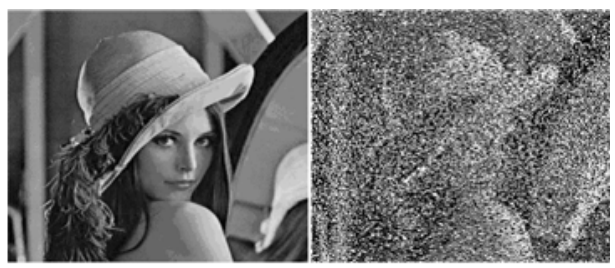

Fig 4.(a)origanal Image (b) Corrupted With 60\% Noise

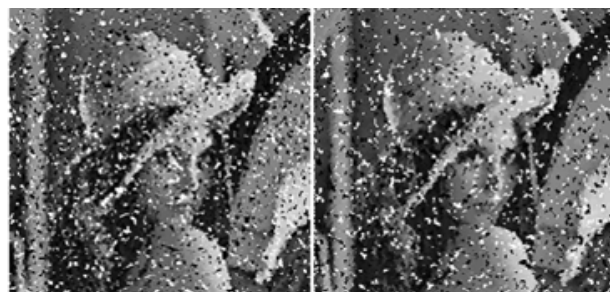

Fig 4.(c)Output from Median Filter (d)output From Cwm Filter

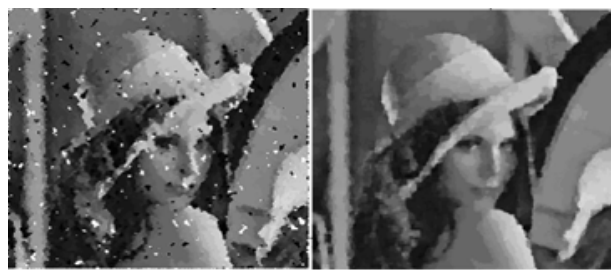

Fig 4.(e)Output from Pwmad Filter(f) Output From Ocs Filter 


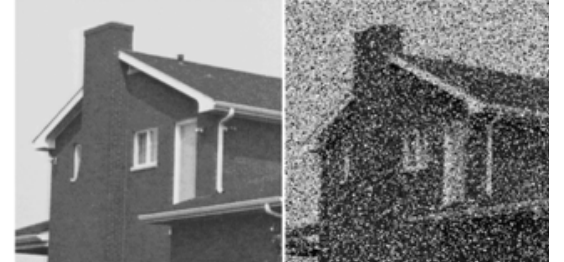

Fig 5.(a)Origanal Image (b)corrupted With 30\% Noise

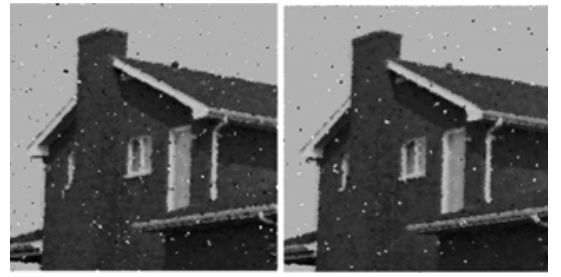

Fig 5.(c)Output from Median Filter (d) Output From Cwm Filter

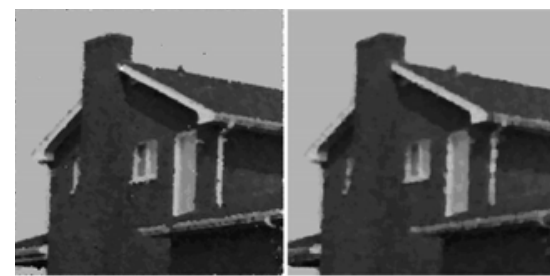

Fig 5.(e)Output from Pwmad Filter (f)output From Ocs Filter

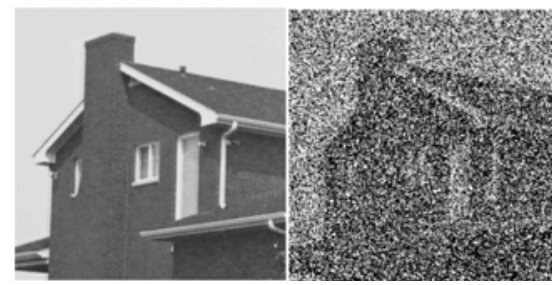

Fig 6.(a)Origanal Image(b) Corrupted With 60\% Noise

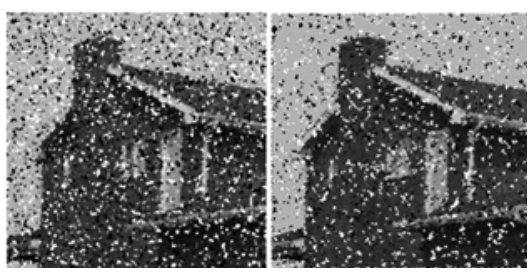

Fig 6.(c)Output from Median Filter (d)output From Cwm Filter

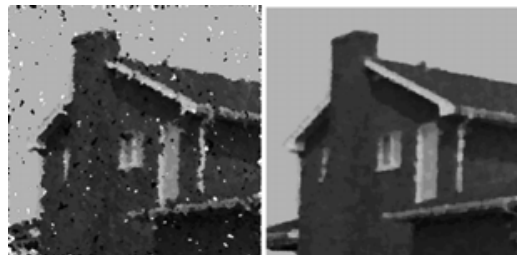

Fig 6. (e)Output from Pwmad Filter (f) Output From Ocs Filter

\section{CONCLUSION}

This paper highlighted the performance of a robust open-close sequence filter. We have proposed a robust open-close sequence filter to remove impulse noise in highly corrupted images. The proposed robust open-close sequence filter is based on mathematical morphology and uses an impulse noise detector. The morphological residue detector powerfully determinates the impulse noise with a low percentage error.

The robust open close sequence filters effectively remove high probability impulse noises. The block smart erase algorithm briefly eliminates the black and white blocks in the filtered image. The simulation results indicate that the proposed filter performances better than other nonlinear filtering techniques and represents robust ability of impulse noise removal.

\section{REFERENCES}

[1] W. K. Pratt, 1975. "Median filtering," Image Proc. Institute, University of Southern California, Los Angeles, Tech. Rep., September.

[2] R.K. Brownrigg, 1984. "The weighted median filter," Communication of ACM, vol. 27 , Issue 8, pp. 807-818.

[3] T. Sun, M. Gabbouj and Y. Neuvo, 1994. "Center weighted median filters: some properties and application in image processing," Signal Processing of ACM, vol. 35, pp. 213-229.

[4] T.Sun, M.Gabb,J. and Y.Neuv, 1992. "Deterministic properties center weighted median filter" Proceeding of 1992 International Conference on Communication Technology, Beijing, China, Sep. 16-18, , pp.06.09.1-06.09.4.

[5] T. Chen and H.Wu, Jan 2001. "Adaptive impulse detection using center-weighted median filters," IEEE Signal Process. Lett., vol. 8, no. 1, pp. 1-3.

[6] V. Crnojevic, V. Senk, and Z. Trpovski, Jul. 2004. "Advanced impulse detection based on pixelwise mad," IEEE Signal Process. Lett., vol. 11, no. 7, pp. 589-592.

[7] E. Abreu, M. Lightstone, S. K. Mitra, and K. Arakawa, Jun. 1996. "A new efficient approach for the removal of impulse noise from highly corrupted images," IEEE Trans. Image Process., vol. 5, no. 6, pp. 1012-1025.

[8] J. Oh and L. F. Chaparro, May 1998. "Adaptive fuzzy morphological filtering of images," in Proc. IEEE Int. Conf. Acoustic, Speech, Signal Processing, vol. 5, pp. 2901-2904. 
[9] R. H. Chan, C.-W. Ho and M. Nikolova, 2005. "Salt-and-pepper noise removal by median-type noise detectors and detail preserving regularization," IEEE Transactions on Image Processing, vol. 14, no. 10, pp. 1479-1485.

[10] P.-E. Ng and K.-K. Ma, 2006. "Aswitching median filter with boundary discriminative noise detection for extremely corrupted images," IEEE Transactions on Image Processing, vol. 15, pp. 1506-1516.

[11] A. Bovik, 2000. Handbook of Image and Video Processing. Academic Press.

[12] Z. Wang and D. Zhang, 1999. "Progressive switching median filter for the removal of impulse noise from highly corrupted images, "IEEE Transactions on Circuits and Systems II," vol. 46, pp. 78-80.

[13] Shyam Lal, Gaurav Saxena \& Akhilesh R Upadhyay, March 02-03, 2007. "Analysis of Digital Images with Morphological Technique", Proc.Technologia-2007, MPCCET Bhilai, Chhattisgarh-India.

[14] J. Serra, Image, 1988. "Analysis and Mathematical Morphology", Academic Press, New York.

[15] J. Song and E. Delp, Jun. 1990. "The analysis of morphological filters with multiple structuring elements," Comput. Vis. Graph., Image Process., vol. 50, pp. 308-328.

[16] S. Mukhopadhysy and B. Chanda, 2002. "An edge preserving noise smoothing technique using multi-scale morphology," Signal Processing of ACM, vol. 82, pp. 527-544.
[17] S. Zhang and M. A. Karim, Nov. 2002. "A new impulse detector for switching median filters," IEEE Signal Process. Lett., vol. 9, no. 11, pp. 360-363.

[18] Deng Ze Feng, Yin Zhou Ping, and Xiong You Lou. Jan. 2007. "High probability impulse noise removing algorithm based on mathematical morphology", IEEE Signal Process. Lett., vol. 14, no. 1, pp. 31-34.

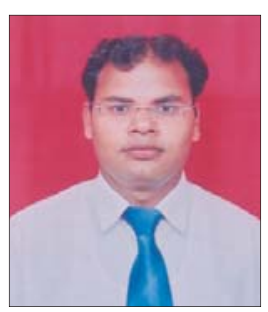

Shyam Lal has been Assistant Professor in Department of Electronics \& Communication Engineering, Moradabad Institute of Technology, Moradabad (U.P)-India. He has published more than 22 papers in the area of Digital Image Processing and Wireless

Communication \& Computing at National/International Level

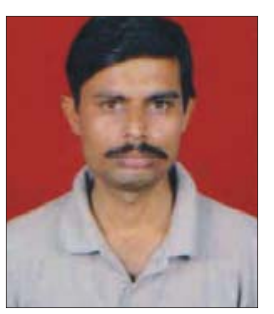

Akhilesh Shukla has been Senior Lecturer in Department of Electronics \& Communication Engineering, Moradabad Institute of Technology, Moradabad (U.P.)-India. He has published more than 12 papers in the area of Microwave, Digital Image Processing and Wireless Communication \& Computing at National/International Level. 